\title{
ESTUDIOS
}

\section{A los veinte años de "Populorum Progressio"}

"El desarrollo integral del hombre no puede darse sin el desarrollo solidario de la Humanidad" (Populorum Progressio, n. ${ }^{\circ} 43$ ).

\section{Por Javier GOROSQUIETA}

El 26 de marzo de 1967, domingo de Pascua, el papa Pablo VI ponía su firma al pie de la enciclica "Populorum Progressio" (PP). La Iglesia tenia ya una doctrina coherente y universal sobre el desarrollo. El desarrollo, para ser auténtico, habria de ser integral, de todo el hombre, y solidario, de toda la Humanidad (Cfr. PP, n. ${ }^{\circ} 5$ ).

Han pasado veinte años y la encíclica no ha envejecido. Conserva, por el contrario, toda su frescura y todo su vigor. Es verdad que PP fue escrita en un contexto de fuerte desarrollo de la economía occidental, incluso de la economia mundial. Durante los años sesenta el comercio mundial fue creciendo, efectivamente, a un ritmo del 8 por 100 anual. Fueron veintidós años de prosperidad permanente $y$ creciente, los que van desde el fin de la segunda postguerra mundial, en 1951, hasta el comienzo de la actual crisis económica en 1973. Un desarrollo, como digo, continuado, aunque con ligeras oscilaciones. Esa larga oleada de prosperidad se intetrumpe bruscamente en 1973, y pasa al primer plano de las preocupaciones socioeconómicas mundiales el problema de la crisis.

Pero PP no ha perdido, en absoluto, actualidad. En primer lugar, porque en estos últimos trece años de crisis las diferencias entre paises ricos y países pobres, lejos de haberse aminorado, han aumento todavía. En segundo término, porque ya se adivina en el horizonte internacional la salida de la crisis económica por parte de los países industriales y la vuelta a su normalidad económica y a su ulterior desarrollo, aunque con grandes variantes, p. ej., en lo relativo al empleo y al ocio. Son, en efecto; cada vez más los expertos que piensan que la era del pleno empleo en sentido tradicional ha pasado a la historia y que es preciso configurar una nueva sociedad del ocio, del tiempo libre y de menor volumen global de trabajo necesario. Estamos, por tanto, en relación con el desarrollo, en situación muy parecida a la de 1967. Por eso será oportuno rememorar hoy el núcleo de las enseñanzas de PP. 


\section{Javier Gorosquieta}

\section{DESARPOLLO INTEGRAL}

El desarrollo integral del hombre consiste en la humanización de la persona, es decir, en pasar "de condiciones de vida menos humanas a condiciones más humanas" (PP, n. 20 ). Enumera PP las condiciones menos humanas: "Las carencias materiales de los que están privados del mínimo vital y las carencias morales de los que están mutilados por el egoísmo... Las estructuras opresoras que provienen del abuso del tener o del abuso del poder, de la explotación, de los trabajadores o de la injusticia de las transacciones" (PP n.. 21). Enumera y escalona, de bien en mejor subiendo, las condiciones de vida más humanas de la persona: "el remontarse de la miseria a la posesión de lo necesario, la victoria sobre las calamidades sociales, la ampliación de los conocimientos, la adquisición de la cultura... El aumento en la consideración de la dignidad de los demás, la orientación hacia el espiritu de pobreza, la cooperación en el bien común, la voluntad de paz.. El reconocimiento, por parte del hombre, de valores supremos, $y$ de Dios, que de ellos es la fuente y el fin... Por fin y especialmente: la fe, don de Dios acogido por la buena voluntad de los hombres, y la unidad en la caridad de Cristo, que nos llama a todos a participar, como hijos, en la vida del Dios vivo, Padre de todos los hombres" (PP n. $\left.{ }^{\circ} 21\right)$.

Ahí está, en todo ello, el desarrollo integral para el cristiano y para todo hombre, que está llamado a serlo. En el desarrollo dentro de un Humanismo integral abierto a la transcendencia, abierto a Dios. "Un humanismo cerrado - dice PP en otro lugar-impenetrable a los valores del espíritu y a Dios, que es la fuente de ellos, podría aparentemente triunfar. Ciertamente, el hombre puede organizar la tierra sin Dios, pero al fin y al cabo, sin Dios no puede menos de organizarla contra el hombre. Un humanismo exclusivo - dice citando a De Lubac - es un humanismo inhumano..." ". "El hombre - cita ahora a Pascal- supera infinitivamente al hombre" (PP n. ${ }^{\circ} 42$ ).

Por el contrario, "por su inserción en el Cristo vivo, el hombre tiene el camino abierto hacia un progreso nuevo, hacia un humanismo transcendental, que le da su mayor plenitud; tal es la finalidad suprema del desarrollo personal" (PP n.0 16).

Concibe PP el desarrollo humano, individual y comunitario, como un deber. "El crecimiento humano -escribe - constituye como un resumen de nuestros deberes" (PP n. ${ }^{\circ}$ 16). Tal es, a nuestro juicio, la sustancia del desarrollo integral según PP.

El papa creyó ver ahí la aspiración de los hombres de su tiempo. "Verse libres de la miseria, hallar con más seguridad la propia subsisten- 


\section{A los veinte años de "Populorum Progressio"}

cia, la salud, una ocupación estable; participar todavía más en las responsabilidades, fuera de toda opresión y al abrigo de situaciones que ofenden su dignidad de hombres; ser más instruidos; en una palabra, hacer, conocer y tener más para ser más; tal es la aspiración de los hombres de hoy" (PP n. ${ }^{\circ}$ 6).

Frente a ella, Pablo VI colocaba como contraste la dura realidad: "un gran número de ellos se ven condenados a vivir en condiciones que hacen ilusorio este legítimo deseo" (PP n. ${ }^{\circ}$ 6).

\subsection{Contraste}

Podemos ilustrar este contraste con algunos datos de nuestros días. Las desigualdades, desde 1967, permanecen; más aún, se han incrementado.

Renta Nacional "per cápita" de países ricos en 1980: Suiza, 943.071 ptas; Alemania Federal, 744.293; Dinamarca, 733.733; Suecia, 708.338; Estados Unidos, 646.906; Holanda, 642.779. Renta Nacional "per cápita" de paises pobres: India, 11.650 ptas.; Sri Lanka, 12.858; Pakistán, 16.521; Indonesia, 20.259; Perú, 31.619, y muchos más, parecidos, entre los países pobres (1). Contraste, como vemos, de uno a setenta u ochenta.

La cuarta parte (rica) de la población de la tierra produce, sobre un tercio de la superficie terrestre, el 57 por 100 de los alimentos mundiales. En Brasil muere anualmente un cuarto de millón de niños por falta de alimento. Unos dos millones de personas mueren anualmente de hambre en el mundo. De los 2.500 millones largos de personas en los paises en desarrollo, 500 millones no tienen lo suficiente para comer, están subalimentados; y 1.500 millones no tienen suficientemente bueno para comer; están mal alimentados. 500 millones largos ingieren escasas calorías; 1.500 millones escasas vitaminas, productos minerales y proteínas. $Y$ estas cifras pecan, sin duda, por ser demasiado optimistas.

Los paises industrializados, que comprenden el 26 por 100 de la población controlan: el 68 por 100 de la producción mundial, el 81 por 100 del consumo de energía, el 70 por 100 del consumo de ferti.izantes, el 85 por 100 de los tractores, el 88 por 100 de la producción de hierro y acero, el 87 por 100 de la producción de armas.

La mortalidad infantil, que es casi nula en los países industriales, es todavía del 50 por 1.000 en Sierra Leona y Guinea, del 47 por 1.000 en Somalia, del 39 por 1.000 en Angola, del 36 por 1.000 en Alto Volta. Cifras parecidas se dan en los demás países en vías de desarrollo. 


\section{Javier Gorosquieta}

En cuanto a la esperanza de vida, es sólo de 34 años en Sierra Leona, de 35 en Gambia, de 42 en Angola, Benin, Malí, Alto Volta, de 45 en Malawi y la India, etc.

Analfabetismo: en Africa son todavia analfabetos el 83 por 100 de las mujeres y el 63 por 100 de los varones.

En la India sus habitantes destinan todavía el 60 por 100 de su presupuesto alimentario a cereales, mientras los países de Europa Occidental apenas dedican a cereales el 10 por 100 de ese presupuesto.

Las desigualdades internas son también mayores en los países en vías de desarrollo. Mientras en Alemania la relación del ingreso entre el 20 por 100 de la población más pobre del pais y el 20 por 100 más rico es de 1 a 6 , en Méjico es de 1 a 20 y en Brasil de 1 a 33 . En los países subdesarrollados una gran parte de la renta nacional está, sí, en manos de una minoría. India: el 20 por 100 de la población posee el 55 por 100 de la renta nacional. Sri Lanka: el 20 por 100 de la población dispone del 50 por 100 de la renta nacional. Puerto Rico: el 10 por 100 de la población posee el 41 por 100 de la renta nacional. Colombia: el 2,6 por 100 de la población dispone del 40 por 100 de la renta nacional. Senegal: el 2 por 100 de la población, en buena parte de ascendencia europea, posee el 34 por 100 de la renta nacional. Camerún: el 0,5 de la población, también en su mayoría de origen europeo, posee el 23 por 100 de la renta nacional.

Se podrian multiplicar las cifras. Son desigualdades que representan una gran injusticia objetiva y que demandan, como PP, un desarrollo universal, integral y solidario.

\subsection{Dependencia}

En cuanto a la dependencia de los países pobres respecto de los ricos, podemos distinguirla hoy al menos en cuatro formas o expresiones. En primer lugar la dependencia clásica, la que supone la concepción leninista del imperialismo: un mundo en el que los países subdesarrollados producen materias primas y las exportan al centro económico mundial y el centro produce artículos manufacturados y los exporta a la periferia. Este modelo ha sido particularmente significativo con el imperio británico. Inglaterra fue la pionera de la revolución industrial en Europa y en el mundo. Se adelantó a las demás naciones en el proceso de industrialización. Adquirió así una posición ventajosa para vender sus propias manufacturas. Por otro lado, disponía en su extenso dominio 


\section{A los veinte años de "Populorum Progressio"}

colonial de grandes recursos naturales $y$ de productores de materias primas. Se estableció así un comercio o intercambio de manufacturas británicas contra materias primas coloniales. Ambos lados, pero sobre todo la metrópoli, salian favorecidos con el sistema. La ideología liberal librecambista y cosmopolita ofrecía al mismo un sustrato teórico o filosófico. Las manufacturas británicas no encontraban, en virtud del librecambismo, fronteras u obstáculos aduaneros en las colonias. Este esquema de intercambio y esta forma de dependencia fue la característica y la que predominó hasta 1945. Típica consecuencia de la misma fue el llamado "efecto Prebisch", es decir, el progresivo deterioro de la relación real de intercambio entre materias primas y manufacturas, en perjuicio de las primeras. Cada vez se podían adquirir menos manufacturas con los mismos volúmenes de materias primas. Así se calcula que en los años cincuenta y sesenta los países pobres perdieron por ese "efecto Prebisch" un montante superior a la ayuda recibida en ese mismo lapso de tiempo.

Pero, a partir sobre todo de 1945, van apareciendo otras modalidades más sutiles de dependencia. La primera es la denominada dependencia tecnológica. En buena parte las materias primas naturales, v.gr., el caucho, los abonos nitrogenados, son sustituidos por producciones sintéticas, químicas, con lo que los paises ricos no necesitan ya en buena parte importarlas de los países en vías de desarrollo. Por otra parte, tres décadas de pleno empleo y Estado Benefactor en los países industriales han incrementado significativamente el coste del trabajo, estimulando la inmigración de mano de obra y la emigración de capacidad productiva hacia los países de la periferia. Además, una revolución técnica en los transportes, comunicaciones y sistemas de información, ha permitido al mundo de los negocios distribuir capacidad productiva por todo el mundo para beneficiarse de las condiciones locales a la vez que operan como un todo integrado. Se da también el fenómeno que presenta la versión más simple del imperialismo de Marx: el capitalismo central invierte en la periferia a fin de poder reducir la composición orgánica del capital y aumentar con ello la tasa de ganancia.

Toda esta extroversión del capital hacia la periferia, por diferentes motivos, va acompañada necesariamente de una inmensa exportación de tecnología, habitualmente descubierta y patentada en los países centrales. A través de la misma es como se produce el control y la dependencia tecnológicos. Es esta capacidad de generar un proceso autoalimentado de dependencia tecnológica y sus repercusiones en la repartición del excedente y en el control del desarrollo de las fuerzas productivas en la periferia lo que parece contener la esencia del novísimo carácter de la dependencia. Con la particularidad de que muchas veces se da una ina- 


\section{Javier Gorosquieta}

decuación entre la tecnología importada, en concreto por las multinacionales, y las disponibilidades de recursos propios en el pais receptor.

Tal ha sido, por ejemplo, el caso del maiz y de la soja en la fabricación de piensos compuestos en España. Se desarrolló fuertemente en nuestro país la ganadería industrial en la década de los sesenta. Pero se desarrolló a través de una industria de piensos compuestos inducida por las multinacionales; una industria que adoptó como materias primas básicas el maíz y la soja, en los que la producción nacional es absolutamente insuficiente. Consecuencia: una fuerte dependencia tecnológica de la industria americana de piensos compuestos, que eleva las importaciones de maíz y de soja americanos a ser los renglones más voluminosos de nuestra balanza de mercancias.

Con frecuencia la tecnología importada por las multinacionales reduce la fuerza de trabajo necesaria, utiliza intensivamente el factor capital e incrementa el consumo de energía. De ello se derivan consecuencias negativas en países con situaciones endémicas de paro, subempleo y emigración, escasez de recursos financieros y problemas en sus balanzas de pagos. Por atra parte, el tipo de compromisos que unen a los fabricantes con las casas matrices multinacionales, implica a menudo restricciones a la libre exportación de los productos licenciados y fuertes pagos por las diferentes modalidades de transferencia de tecnología.

Una tercera forma de dependencia es la que consiste en el esfuerzo por especializar al Tercer Mundo en la producción industrial clásica (siderurgia, industria textil tradicional, construcción naval, etc.), incluida, por lo tanto, la producción de bienes de capital, mientras que el centro se reserva para sí las ramas ultramodernas de actividad (automoción, electrónica, informática, telemática, bioingeniería, la conquista del espacio, la energía nuclear, etc.). Esto lleva a un más elevado grado de cualificación media del trabajo en el centro respecto de la periferia $y$, por lo tanto, a una nueva modalidad de dependencia que podriamos denominar profesional.

La cuarta forma de dependencia es la que visualiza Hymer en su conocido artículo "Las Empresas Multinacionales y la Ley del Desarrollo Desigual". El autor ve un mundo futuro posible en el que "un régimen de corporaciones multinacionales noratlánticas tenderia a producir una división jerárquica del trabajo en regiones geográficas, correspondientes a la división vertical del trabajo dentro de la firma. Tendería a centralizar las ocupaciones de toma de decisiones a alto nivel en unas cuantas ciudades clave de los países avanzados, rodeadas de varias subcapitales regionales, $y$ a condenar al resto del mundo a niveles más bajos de actividad e ingresos, esto es, a la posición relativa de pueblos y aldeas del 
A los veinte años de "Populorum Progressio"

nuevo sistema imperial" (1). Un nuevo imperio, pues, a través de la transnacionalización del capitalismo, con un régimen de grandes decjsiones centrales y centralizadas barriendo para la casa noratlántica.

Por todos estos sutiles caminos de la desigualdad y la dependencia el capitalismo como sistema, tanto del Oeste como del Este, colabora, por un lado, al progreso económico global, pero a través de novísimas injusti cias y modalidades de dominación.

\subsection{Desequilibrio creciente}

Ya lo dice PP: "El desequilibrio crece: unos producen con exceso géneros alimenticios que faltan cruelmente a otros, y estos últimos ven que sus exportaciones se hacen inciertas" (PP n. $\left.{ }^{\circ} 8\right)$.

Efectivamente: la crisis agraria típica de los países industriales no es una crisis de escasez, sino de sobreabundancia, una crisis de excedentes. Montañas de excedentes de mantequilla, de leche en polvo, de otros productos lácteos, de carne, etc., se amontonan, mientras en el Sahel y otras regiones del mundo se mueren de hambre. ¿Por qué no regalar los excedentes? Porque, como dicen, simplemente el hambre no es demanda de alimentos en términos económicos. Solamente lo es el hambre solvente. Entonces se podrían entregar los excedentes en forma de crédito, en buenas condiciones de plazo e interés, al desarrollo. El éxito, técnicamente asesorado, de este último posibilitaría la devolución de aquellos préstamos en especie.

Reconocía Pablo Vl que el desarrollo es una realidad ambigua.. "Todo crecimiento -escribe- es ambivalente. Necesario para permitir que el hombre sea más hombre, lo encierra como en una prisión, desde el momento que se convierte en el bien supremo, que impide mirar más allá. Entonces los corazones se endurecen y los espíritus se cierran; los hombres ya no se unen por amistad, sino por interés, que pronto les hace oponerse unos a otros y desunirse. La búsqueda excesiva del poseer se convierte en un obstáculo para el crecimiento del ser $y$ se opone a su verdadera grandeza; para las naciones, como para las personas, la avaricia es la forma más evidente de un subdesarrollo moral" (PP n. ${ }^{\circ} 19$ ).

Como decia Gandhi: "el mundo tiene recursos para cubrir las necesidades de todos, no para satisfacer la codicia universal".

(1) Carlos FORTiN en Osvaldo Sunkel y Varios, "Transnacionalización y Dependencia", Ediciones Cultura Híspánica, Madrid 1980, pág. 183 ss. 
Javier Gorosquieta

\subsection{Acciones que emprender}

Puesto que el Papa escribia en PP para impulsar a la acción, señala en esta parte sobre el desarrollo integral algunas acciones que se deberian emprender:

Primera: ordenar la propiedad. "La tierra - dice - ha sido dada para todo el mundo y no solamente para los ricos. Es decir,... la propiedad privada no constituye para nadie un derecho incondicional y absoluto. No hay ninguna razón para reservarse en uso exclusivo lo que supera la propia necesidad, cuando a los demás les falta lo necesario. En una palabra: el derecho de propiedad no debe jamás ejercitarse con detrimento de la utilidad común"' (PP n. 23).

Segunda: promover la industrialización, de la que tiene la encíclica un concepto muy positivo: "Necesaria para el crecimiento económico -afirma - y para el progreso humano, la industrialización es al mismo tiempo señal y factor del desarrollo. El hombre, mediante la tenaz aplicación de su inteligencia y de su trabajo, arranca poco a poco sus secretos a la Naturaleza, y hace un uso mejor de sus riquezas. Al mismo tiempo que disciplina sus costumbres, se desarrolla en él el gusto por la investigación y la invención, la aceptación del riesgo calculado, la audacia en las empresas, la iniciativa generosa y el sentido de responsabilidad" (PP n. $\left.{ }^{\circ} 25\right)$.

Tercera: acertar con el sistema económico. Vamos a detenernos algo en este apartado. Ni liberalismo individualista, ni colectivismo socialista; sí programación no arbitraria, contando con los cuerpos intermedios, con las iniciativas sociales.

"La sola iniciativa individual -enseña- y el simple juego de la competencia no serían suficientes para asegurar el éxito del desarrollo. No hay que arriesgarse a aumentar todavía más la riqueza de los ricos y la potencia de los fuertes, confirmando así la miseria de los pobres y añadiéndola a la servidumbre de los oprimidos. Los programas son necesarios para 'animar, estimular, coordinar, suplir e integrar' la acción de los individuos y de los cuerpos intermedios. Toca a los poderes públicos escoger y ver el modo de imponer los objetivos que hay que proponerse, las metas que hay que fijar, los medios para llegar a ellas, estimulando al mismo tiempo todas las fuerzas, agrupadas en esta acción común. Pero ellas han de tener cuidado de asociar a esta empresa las iniciativas privadas y los cuerpos intermedios. Evitarán así el riesgo de una colectivización integral o de una planificación arbitraria que, al negar la libertad, excluiría el ejercicio de los derechos fundamentales de la persona humana" (PP n.० 33). 
A los veinte años de "Populorum Progressio"

Se apunta, pues, como vemos, una especie de tercera vía entre capitalismo liberal y colectivismo socialista.

Sobre el capitalismo liberal extremo PP tiene tal vez el juicio más duro existente en la Doctrina Social de la Iglesia en esta materia. Dice en el n. ${ }^{\circ}$ 26: "Pero, por desgracia, sobre estas nuevas condiciones de la sociedad ha sido construido un sistema que considera el provecho como motor esencial del progreso económico, la concurrencia como ley suprema de la economía, la propiedad privada de los medios de producción como un derecho absoluto, sin limites ni obligaciones sociales correspondientes. Este liberalismo sin freno, que conduce a la dictadura, justamente fue denunciado por Pío XI como generador del "imperialismo internacional del dinero". No hay mejor manera de reprobar un tal abuso que recordando solemnemente, una vez más, que la economía está al servicio del hombre. Pero si es verdadero que un cierto capitalismo ha sido la causa de muchos sufrimientos, de injusticias y luchas fratricidas, cuyos efectos duran todavía, sería injusto que se atribuyera a la industrialización misma los males que son debidos al nefasto sistema que la acompaña. Por el contrario, es justo reconocer la aportación irreemplazable de la organización del trabajo y del progreso industrial a la obra del desarrollo".

La solución, para PP, dentro de un régimen de libertad: la política de reformas, audaces, innovadoras, urgentes. "El desarrollo exige -asevera-transformaciones audaces, profundamente innovadoras. Hay que emprender, sin esperar más, reformas urgentes. Cada uno debe aceptar generosamente su papel, sobre todo los que por su educación, su situación y su poder tienen grandes posibilidades de acción" (PP n.*32).

Esta misma vía de reformas, de participación, encontrará una continuidad en 1981 con la encíclica Laborem Exercens de Juan Pablo Il. También ahí se descalifican dos tipos de capitalismo: el que llama "capitalismo rígido" o liberal extremo y el único capitalismo realmente existente como sistema mundial de economía y de poder. También ahí se encuentra la única tabla de salvación ética para el mismo en el capitàlismo reformista (2).

En Laborem Exercens se descalifica asimismo éticamente al colectivismo socialista realmente existente. Pero se admite una via de evolución y de legitimación ética positiva posible del mismo, desde el propio colectivismo; es de nuevo el camino de la socialización y la vitalidad de

(2) Véase Javier GOROSOUIETA: El capitalismo en "Laborem Exercens", en "Revista de Fomento Social", n. ${ }^{\circ}$ 146, abril-junio 1982, págs. 145 a 154. 


\section{Javier Gorosquieta}

todo género de sociedades intermedias suficientemente autónomas a nivel de sociedad (3).

\subsection{Promoción cultural}

En su primera parte PP da una gran importancia a la promoción cultural como componente básico del desarrollo integral. Educación básica o, lo que es lo mismo, alfabetización, por un lado, y ulterior elevación cultural, por otro.

De la primera dice: "Se puede también afirmar que el crecimiento económico depende en primer lugar del progreso social; por eso la educación básica es el primer objetivo de un plan de desarrollo. Efectivamente, el hambre de instrucción no es menos deprimente que el hambre de alimentos: un analfabeto es un espíritu subalimentado. Saber leer y escribir, adquirir una formación profesional, es recobrar la confianza en sí mismo y descubrir que se puede progresar al mismo tiempo que los demás" (PP n. $\left.{ }^{\circ} 35\right)$.

En el tema de la ulterior promoción cultural, PP resalta la conveniencia de mantener la identidad, a veces muy rica, de la civilización específica de los países pobres. "Rico o pobre - indica-cada país posee una civilización recibida de sus mayores: instituciones exigidas por la vida terrena y manifestaciones superiores -artísticas, intelectuales y religiosas- de la vida del espíritu. Mientras que éstas contengan verdaderos valores humanos, seria un grave error sacrificarlas a aquellas otras. Un pueblo que lo permitiera perdería con ello lo mejor de símismo y sacrificaría, para vivir, sus razones de vivir. La enseñanza de Cristo vale también para los pueblos: '¿De qué le sirve al hombre ganar todo el mundo si pierde su alma?" (PP n. $\left.{ }^{\circ} 40\right)$.

\section{DESARROLLO SOLIDARIO}

El desarrollo solidario de toda la Humanidad es, para PP, parte integrante del desarrollo integral. "El desarrollo integral del hombre - diceno puede darse sin el desarrollo solidario de toda la Humanidad" (PP $\left.n^{\circ} 43\right)$.

(3) Véase Javier GOROSOUIETA: ¿Continuidad o profunda novedad", en "Sal Terrae", núm. 824 , enero 1982, págs. 3 a 17 . 


\section{A los veinte años de "Populorum Progressio"}

Hay, en esta parte, como tres grandes capitulos. Primero: "deber de solidaridad en la ayuda que las naciones ricas deben aportar a los países en vías de desarrollo". Segundo: "deber de justicia social, enderezando las relaciones comerciales defectuosas entre los pueblos fuertes y débiles". Tercero: "deber de caridad universal, por la promoción de un mundo más humano para todos, en donde todos tengan que dar y recibir, sin que el progreso de los unos sea un obstáculo para el desarrollo de los otros" (PP n. ${ }^{\circ} 44$ ).

\subsection{Deber de solidaridad}

Sobre el deber de solidaridad PP cita al Concilio: "los pueblos ya desarrollados tienen la obligación gravísima de ayudar a los países en vías de desarrollo" (PP $\mathrm{n}^{\circ} 48$ ). Y añade: "Ante la creciente indigencia de los países subdesarrollados, se debe considerar como normal el que un pais desarrollado consagre una parte de su producción a satisfacer las necesidades de aquéllos; igualmente normal que forme educadores, ingenieros, técnicos, sabios que pongan su ciencia y su competencia al servicio de aquéllos" (PP n. ${ }^{\circ} 48$ ).

En relación con este tema conocemos cómo la Primera Década de las Naciones Unidas para el Desarrollo, 1960, propuso una ayuda a fondo perdido a los países en vías de desarrollo, de un $0,7 \%$ de los respectivos Productos Nacionales Brutos de los países industriales o ricos. Sólo superan, en 1982, ese porcentaje de ayuda, el conjunto de los países de la OPEP con un $1,46 \%$, Holanda con un $1,08 \%$, Noruega con el $1,01 \%$, Suecia con el $1,02 \%$, Dinamarca con el 1,1\%. Dentro de la OPEP destacan Arabia Saudí con el $4,77 \%$, los Emiratos Arabes Unidos con el $2,88 \%$, Kuwait con el $1,98 \%$. El resto de los paises industriales no alcanza el $0,7 \%$ establecido por la ONU. La media de aportación, por el contrario, es sólo del $0,39 \%$. Queda, por lo tanto, mucho camino por recorrer en ese sentido (4).

PP apunta más arriba que la ONU. A saber, aplica la doctrina moral clásica sobre los bienes superfluos individuates a los bienes superfluos de las sociedades ricas. "Lo superfluo -escribe- de los países ricos debe servir a los países pobres. La regla que antiguamente valia en favor de los más cercanos debe aplicarse hoy a la totalidad de las necesidades del mundo" (PP n. $\left.{ }^{\circ} 49\right)$.

(4) Véase Leelananda DE SILVA: Ayuda al Desarrollo, lepala Editorial, Madrid 1985 , pág. 44. 


\section{Javier Gorosquieta}

PP propuso, además, iniciativas concretas de ayuda, como la creación del que llamó Fondo Mundial para el desarrollo. Sería alimentado con una parte de los gastos militares y tendría como finalidad ayudar a los más necesitados. Se han construido desde 1967 diversas formas de ayuda, pero ese Fondo Mundial no se ha creado todavia (Cfr. PP n. ${ }^{\circ} 51$ ).

\subsection{Deber de justicia}

El problema de la equidad en las relaciones comerciales continúa hoy en pleno vigor. Ya he aludido antes al denominado "efecto Prebisch". Es verdad que durante los primeros años de la crisis hubo un respiro para los países pobres. Se elevaron fuertemente los precios del petróleo y de las materias primas, de tal forma que en sólo año y medio mejoró la relación real de intercambio a favor de los países pobres en torno a un $20 \%$. Pero ahora estamos de nuevo en el punto de partida, con la agravante de que, movidos en parte por aquella euforia de los precios de la energía y materias primas, muchos países en desarrollo se endeudaron fuertemente, lo que ha originado el grave problema actual de la deuda internacional. El conjunto de los países en desarrollo debe actualmente en torno a los 700.000 millones de dólares.

Tampoco hoy, por lo tanto, como en tiempos de PP, sirve el liberalismo individualista como regulador de la justicia en las relaciones comerciales. Son precisos convenios internacionales, acuerdos sobre productos, acuerdos multilaterales, preferencias generalizadas, asociaciones para el desarrollo como la de la Convención de Lomé y otras.

\subsection{Deber de caridad universal}

El deber de caridad universal lo concretaba PP en el deber de solidaridad para con los extranjeros (del Tercer Mundo) y trabajadores emigrantes (PP n.os 67 y 69), en el sentido social de los hombres de negocios occidentales que trabajan en los países pobres (PP n. ${ }^{\circ} 70$ ), en la multiplicación de las misiones de desarrollo (PP n. $\left.{ }^{\circ} 71\right)$ y de los técnicos en servicio social (PP n. $\left.0^{0} 72\right)$.

Otro punto en el que, como en todos los anteriores, $\mathrm{PP}$ sigue siendo de plena actualidad es en el de su pronunciamiento a favor de una autoridad mundial, con el fin, precisamente, de lograr más fácilmente un desarrollo solidario. Decía: "Esta colaboración internacional a vocación mundial requiere unas instituciones que la preparen, la coordinen y la rijan hasta constituir un orden jurídico universalmente reconocido. De 


\section{A los veinte años de "Populorum Progressio"}

todo corazón Nos alentamos las organizaciones que han puesto mano en esta colaboración para el desarrollo, $y$ deseamos que crezca su autoridad: 'Vuestra vocación, dijimos a los representantes de las Naciones Unidas en Nueva York, es la de hacer fraternizar no solamente a algunos pueblos, sino a todos los pueblos (...). ¿Quién no ve la necesidad de llegar así, progresivamente a instaurar una autoridad mundial que pueda actuar eficazmente en el terreno juridico y en el de la política?" (PP n. $\left.{ }^{\circ} 78\right)$.

También hoy, como entonces, hemos de ser favorables a la expropiación en determinadas circunstancias, por razones de interés general. "El bien común - nos señalaba PP - exige algunas veces la expropiación, si, por el hecho de su extensión, de su explotación deficiente o nula, de la miseria que de ello resulta a la población, del daño considerable producido a los intereses del país, algunas posesiones sirven de obstáculo a la prosperidad colectiva" (PP n. $\left.{ }^{\circ} 24\right)$.

También hoy, como entonces, hemos de ser contrarios a la fuga insolidaria de capitales: "Desde luego, no se podría admitir que ciudadanos provistos de rentas abundantes, provenientes de los recursos y de la actividad nacional, las transfiriesen en parte considerable al extranjero, por puro provecho personal, sin preocuparse del daño evidente que con ello infligirian a la propia patria" (PP n. $\left.{ }^{\circ} 24\right)$.

Finalmente digamos que PP tiene un alto concepto del trabajo como continuación de la obra del Creador. "Ya sea él artista o artesano -afirma-, patrono, obrero o campesino, todo trabajador es un creador. Aplicándose a una materia, que se le resiste, el trabajador le imprime un selto, mientras que él adquiere tenacidad, ingenio y espiritu de invención. Más aún, viviendo en común, participando de una misma invención, de un sufrimiento, de una ambición y de una alegría, el trabajo une las voluntades, aproxima los espírtus y funde los corazones; al realizarlo, los hombres descubren que son hermanos" (PP n. $\left.{ }^{\circ} 27\right)$.

\section{PRECEDENTE Y CONTINUIDAD}

PP tiene un precedente inmediato en la Constitución Gaudium et Spes del Concilio Vaticano II. Esta Constitución es del 7 de diciembre de 1965, de poco más de un año antes de PP. Encontramos el precedente no tanto en el apartado sobre la vida económico-social, que trata más bien de los problemas de justicia internos a las economías nacionales, sobre todo de los países industriales, sino en el capítulo $V$, "La comuni- 
Javier Corosquieta

dad de los pueblos", sección 2." "Edificar la comunidad internacional".

PP tuvo una continuidad sobre todo en la declaración del Sínodo Universal de los Obispos de 1971, sobre "La justicia en el mundo", y en Laborem Exercens.

No hay contradicción entre estos cuatro documentos básicos de la Doctrina Social de la Iglesia de estos últimos veintidós años, sino armonía, cohesión, complementariedad y progreso.

Esperemos que PP, joven después de sus primeros veinte años de vida, siga orientándonos para lograr un desarrollo pleno de la persona $y$ un desarrollo solidario de todos los pueblos. 